\title{
A Study of Prehistoric Cloud and Water Texture Human Face Rock Paintings in the Upper Laohahe River
}

\author{
Wu Jiacai \\ North China Rock Art Research Institute, Hongder College, Inner Mongolia Normal University, Hohhot, China \\ Email address: \\ 2378113914@qq.com \\ To cite this article: \\ Wu Jiacai. A Study of Prehistoric Cloud and Water Texture Human Face Rock Paintings in the Upper Laohahe River. American Journal of Art \\ and Design. Vol. 4, No. 1, 2019, pp. 1-7. doi: 10.11648/j.ajad.20190401.11
}

Received: August 22, 2018; Accepted: September 21, 2018; Published: June 4, 2019

\begin{abstract}
To understand the ancient rock paintings correctly, we should start with the analysis of the rock painting chronology and the rock painting form. For the rock paintings rich in quartz crystal, such as the water texture Rock Paintings of Hongshan Hou in the upper reaches of Laohahe River in northern China, we have succeeded in the rock paintings of Hongshan Hou and Zhang Yingrui of Jiguo in Yuan Dynasty according to the dating equipment, procedures and methods used by the international rock painting organizations [1-3]. The tombstone, the tombstone of Zhang Chonglu, the metropolitan governor of the Yuan Dynasty, the tombstone of Confucianism on Quanning Road in the Yuan Dynasty, and other chronologically rich in quartz crystals, were scientifically and noninvasively extracted from the micro-corrosion (microscopic) sample data. According to the corrosion width (area) of quartz crystals in chronological years, the corrosion width per thousand years was obtained, and the production age of rock paintings was calculated. In order to test the accuracy of micro-corrosion dating method, we can adopt the method of cultural relics analogy to make a comparative study on the shape system of rock paintings and the decorative patterns of painted pottery unearthed from rock paintings sites. Results The micro-corrosion dating method was used to measure the E5000+1071/-1071 years and the E5000 years of the moist rhyolite carving in Hongshan Houcao cave. This method was basically consistent with the 5000 years of the colored pottery (carbon 14 dating) unearthed from the rock painting site. Conclusion: Three moist-water rhyolite paintings were found in a river in the upper reaches of Laoha River. The method of making them is the same as that of making them in the same time. The style of making them is the same as that of "cloud and water" in oracle bone inscriptions.
\end{abstract}

Keywords: Cloud and Water Texture, Human Face Rock Paintings, Red Mountain Behind, China

\section{The Geographical Location of Cloud and Water Texture}

In the investigation of northern rock paintings, up to now, we have discovered that there are three areas in the cloud and water texture human Face Rock Paintings, two of which are located in the excavation site of Xiajiadian Lower Ancient City on the north bank of the Yinhe River in Songshan District, Chifeng City, Inner Mongolia; At an altitude of 693 meters, longitude 118.620; latitude 42.369. The other is located at the half-waist of the excavation site of Hongshan Mountain on the east bank of the Yingjin River in the upper reaches of the Laoha River in Hongshan District, Inner Mongolia. The altitude is 664.2 meters, longitude 118.987, and latitude 42.302 .
Three shop rock paintings and red rock paintings are located in the Yin River - Yingjin River - Laoha River - Liaohe river system, the two lines of moire face rock painting straight line distance 31 kilometers (Figure 1).

\section{The Main Shape of Rock Art and Its Surrounding Remains}

\subsection{The Post-Red Mountain}

The post-Red Mountain rock painting is carved by the ancients on the cliffs of red granite. The figure of the cloud and water texture human Face Rock Paintings is $38 \mathrm{~cm}$ high and $40 \mathrm{~cm}$ wide. The groove width is $1-1.8 \mathrm{~cm}$ and the depth is $0.5-1 \mathrm{~cm}$. Wait. The cloud and water texture human Face Rock Paintings is composed of two parts: the above is composed of 
a pair of three-circle swirling patterns. It may seem to be a cautious word of the cloud in the "Shuo Wen Jie $\mathrm{Zi}$ " Oracle bones; the following is composed of three water ripples, which may seem cautious The words of the water in Oracle's "Shuo Wen Jie Zi". The Swedish sinologist Cecilia Lindquist (Chinese name Lin Xili) put together a variety of writings in water, rivers, and prefectures in her "Kingdom of Chinese
Characters" p584[4]. The three characters are related to river water. Water and Kawase are very appropriate in the rock paintings. The structure of the rock paintings is clear and smooth, with smooth and graceful lines. They look far away from the big eyes of a pair of gods and peep into the endless stream of water on the west (Figure 2).

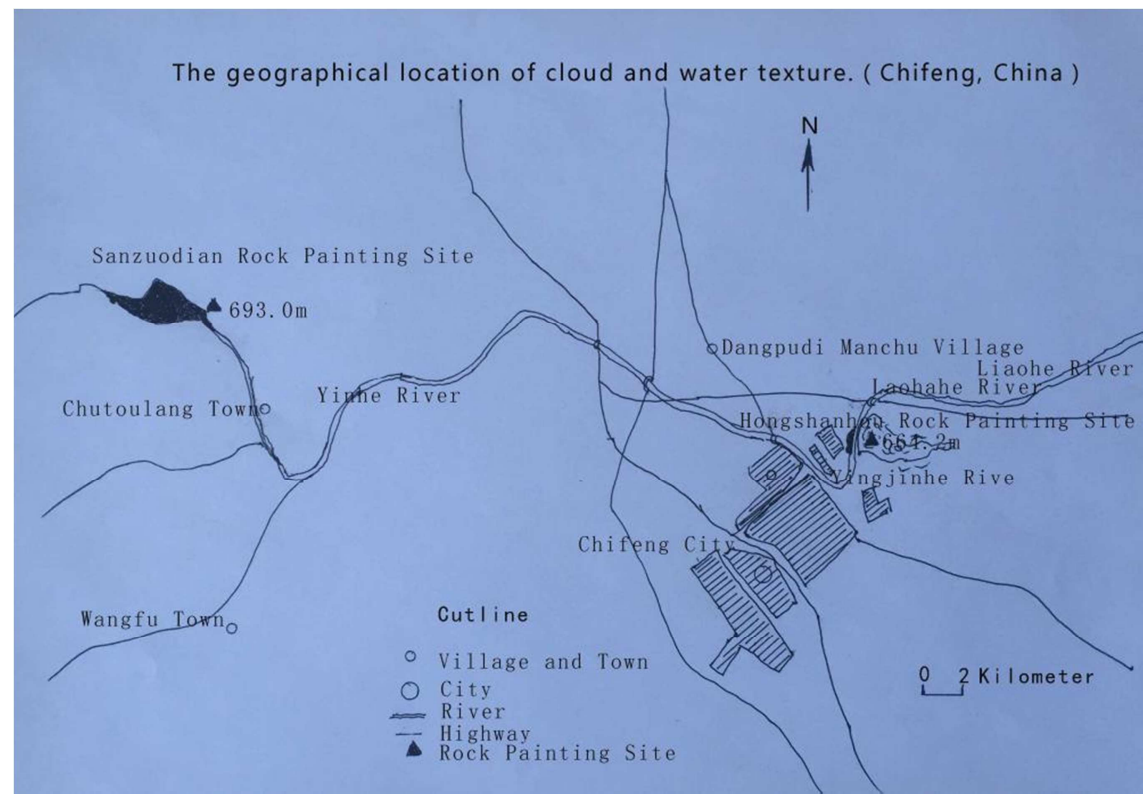

Figure 1. The geographical location of cloud and water texture (Chifeng, China).

The rock paintings exhibiting water vortices are mostly on the banks of the inland rivers and on the circum-Pacific coast. Most of them appear as single vortex patterns, some of which are concentric circles of [5-10] and some of which are vortices [11-16]. The whirlpool painting lines in the double bodies [17-25] and above rotate in the same direction [26, 27], and no symmetric whirlpool-like face rock paintings in the Hongshan cultural area have been found. This posture is unique to the Hongshan cultural region. . However, this special shape was not disclosed in the excavation report of the Xiajiadian Site in detail [28], and was not disclosed in the report on excavated earthenware found in the Hongshan Cultural Site [29].

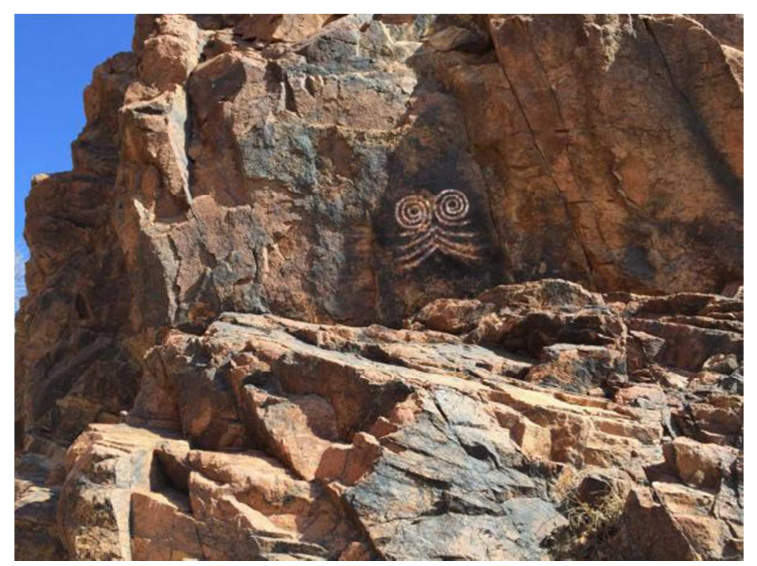

Figure 2. Rock Art Paintings Behind Hongshan Mountain.

There are 11 star-point rock paintings on the red granite platform $50 \mathrm{~cm}$ below the Hongshan Yun Shui Wen Ren Hua Yan rock paintings. They are distributed in the area of the Yangtze River Delta, where the height is $70 \mathrm{~cm}$, the width is 44 $\mathrm{cm}$, and the stars are large. The diameter of $4 \mathrm{~cm}$, the depth of 2 $\mathrm{cm}$, the small diameter of the star hole $2 \mathrm{~cm}$, depth $1 \mathrm{~cm}$, they are arranged in order, is the sky map, or a combination of pictographic symbols, has not yet resolved a satisfactory answer.

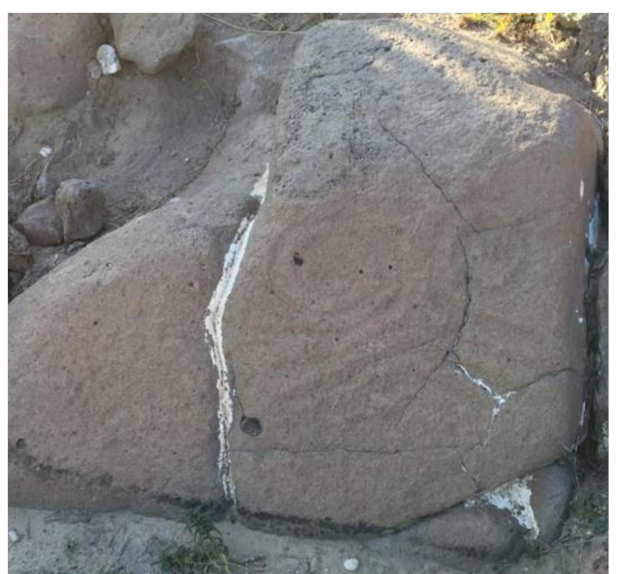

Figure 3. The first spot of cloud and water texture rock paintings in Sanzuodian(place).

\subsection{Three Shop Rock Paintings}

Three shop rock paintings, four rock carvings in total, of which two clouds and water-faced face rock paintings (excluding quartz crystals) face the southeast Yin River. The 
first cloud and water texture rock paintings is carved on the gray sandstone. The rock painting is located on the Xiajiadian lower cultural site wall base. The rock paintings are $48 \mathrm{~cm}$ high and 43 $\mathrm{cm}$ wide. The carving is sharp and the groove width is 1.5 . Centimeter, depth $0.5-1 \mathrm{~cm}$ (Figure 3). The second cloud and water texture rock paintings is 15 meters away from the first rock painting. The rock paintings are carved on the gray sandstone. This cloud and water texture rock paintings is 60 centimeters high and 85 centimeters wide. (Figure 4).

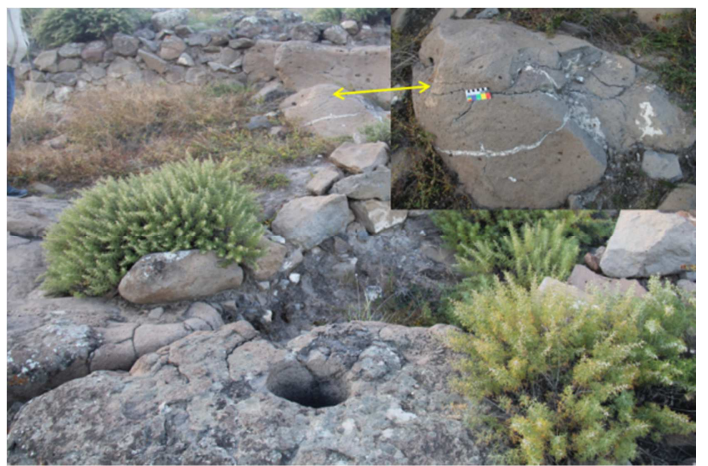

Figure 4. The Second spot of cloud and water texture rock paintings in Sanzuodian(place).
There are two rock carvings on the north side of the cloud and water texture rock paintings: The first cave rock carving is near the first human face rock painting, and the star cave rock face is $129 \mathrm{~cm}$ wide and $90 \mathrm{~cm}$ high, and it is 1.16 square meters. Above the gray sandstone, there are 40 artificially etched stars (Figure 5).

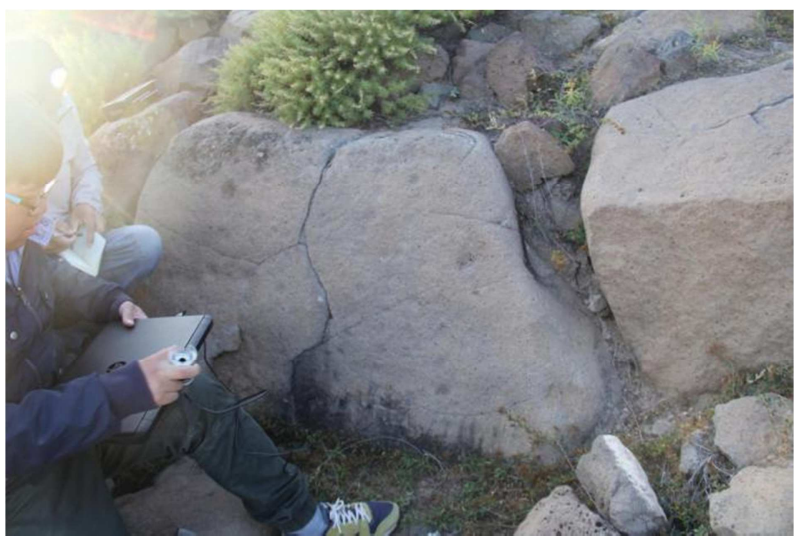

Figure 5. Asterescens rock carving near the cloud and water texture rock paintings in the first location of Sanzuo shop.

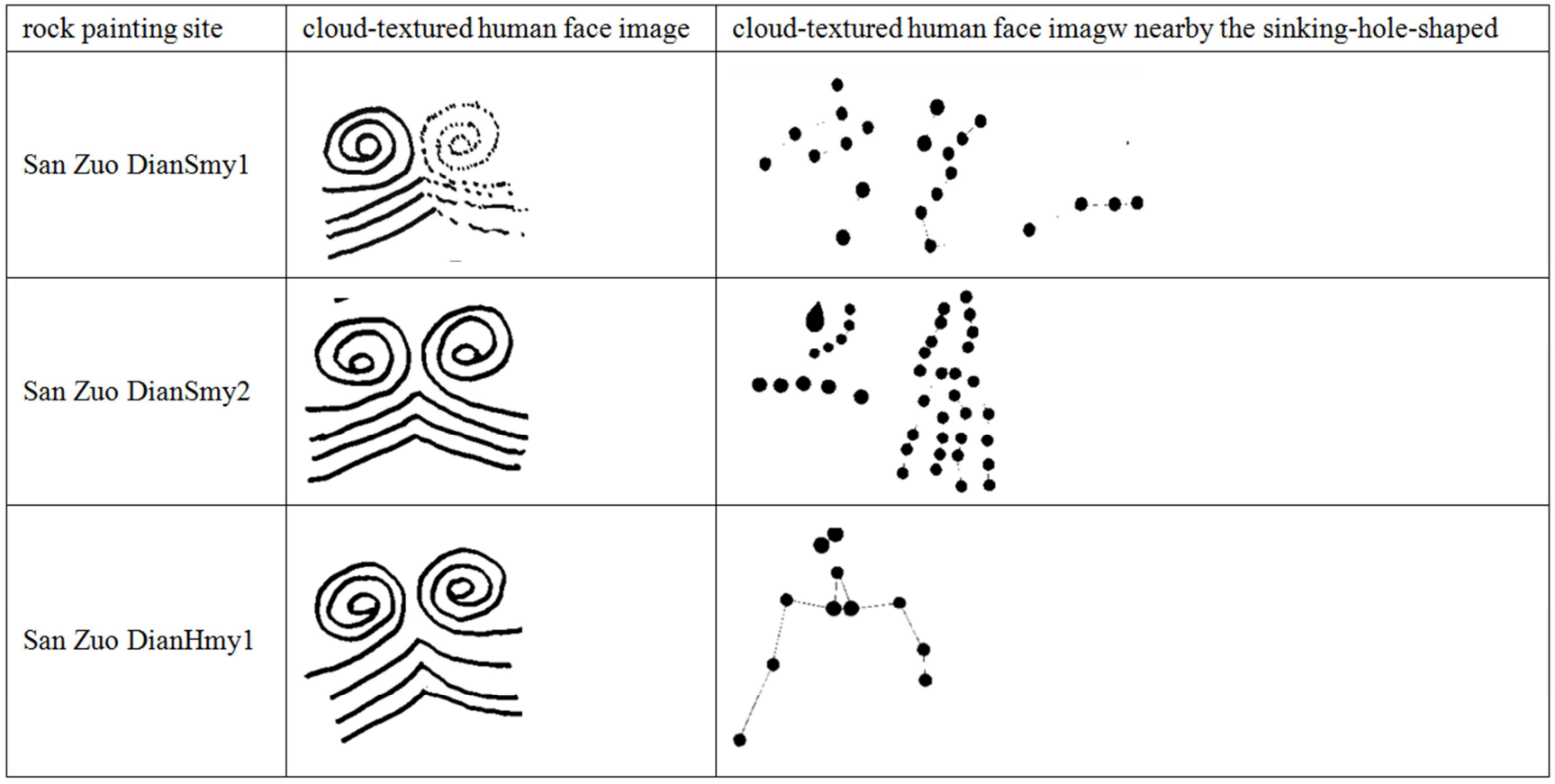

Figure 6. Sanzuodian, the cloud and water texture rock paintings Behind Hongshan Mountain Petrography and Xinguyan Rock Engraving System.

The second starhole was engraved at a distance of $1.5 \mathrm{~m}$ from the north of the second human face rock painting. The starhole was carved on three fractured gray sandstones. The starhole facet was $208 \mathrm{~cm}$ long and $57 \mathrm{~cm}$ high at 1.19 . There are 22 artificially engraved crests on the average limestone sandstone (Figure 6).

\subsection{Hongshanhou Colored Pottery}

The Japanese East Asian Archaeological Society of Hongshan was discovered here from 1935-1938. The excavation report of Chifeng Behind Hongshan did not mention ancient rock paintings. The researchers found many red mountain cultures during the period of Hongshan Culture. The shape of the residual terra cotta was restored to the same shape as the excavation report of [29] of Chifeng Behind Hongshan (Figure 7).

The performance of the cloud and water patterns on rock paintings and pottery means that the ancient people expressed abstract and general representations of their natural environment and resources. The performance of water ripples 
stems from the desire of life for water. Water is the source of life. The survival of human beings cannot be separated from water. The early nomadic peoples had habits and traditions of living by the plants. Not so much "traditions", it would be better to say that making rock art is an indispensable best way to express life and reproduce the race.

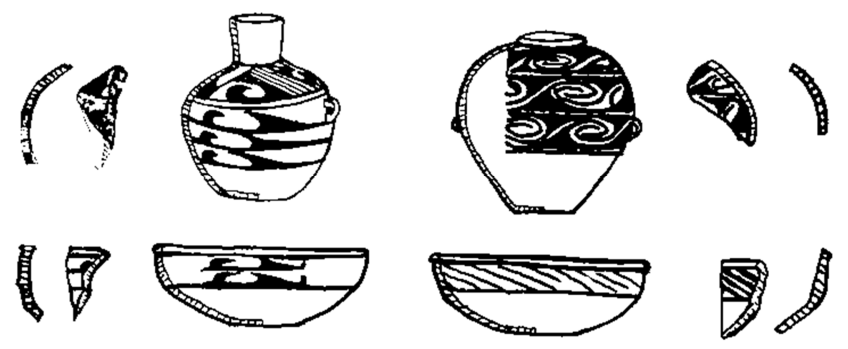

Figure 7. Restoration of colored pottery fragments and artifacts collected from the Hongshan cloud slate rock paintings.

Note; residual pottery film Behind Hongshan rock painting site collection, in the middle of pottery restoration map

The cloud water swirl vortex pattern human face rock painting is currently the first case at home and abroad. The cloud water vortex pattern pottery that appeared in the Yellow River Basin and the West Liaohe River Basin generally follows the same direction as the whirlpool pattern, unlike the Hongshan Mountain. Three shops rock symmetry, the ancient personification of the cloud Shuiwen lines, freehand brushwork, should be the fear of God in the eyes of the ancients. There are no stars in the sky near the awe-inspiring totem, and the three-faced Shuizhuang human face resembles the shape of the rock. The shape of its neighboring stars is different. It should be a description of people's mind-set stars or the sky stars. What is the meaning of it is still unknown.

\section{The Age of Petroglyphs}

The author and according to the dating equipment, procedures and methods used by international rock art organizations (Note 2) succeeded in the post-Hongshan rock paintings, the Yuan Dynasty Jigong Zhang Yingrui gravestones, the Yuan Dynasty chiefs Zhang choulv tombstones, Yuan Dynasty Quanning Road Confucianism monuments. The helium that was rich in quartz crystals was recorded in scientific years, and scientifically no damage was taken to extract macroscopic sample data.

\subsection{Water and Cloud Texture Human-Faced Rock Painting in Hongshanhou}

The Hongshan cloud and water texture rock carving (code name Hmy1.1-1.4) measured four points (Table 1, Figure 8). The water ripple is $18 \mathrm{~cm}$ high, $40 \mathrm{~cm}$ wide, and the groove width is $1.8 \mathrm{~cm}$. At a depth of $1.0 \mathrm{~cm}$, the quartz crystal appears in the groove on the right side of the second water ripple, and there are four crystals with measurable points. The crystal is slightly attenuated at a $90^{\circ}$ angle, and the four-point attenuation is accurately measured. The result is :
Hmy1.1 left oblique vertical point length 56 and width $14 \mu \mathrm{m}$. The Hmy1.2 transverse point is 87 in length and $14 \mu \mathrm{m}$ in width. The vertical point in Hmyl.3 is 187 and $14 \mu \mathrm{m}$ in width. Hmy1.4 right oblique point length 211 , width $14 \mu \mathrm{m}$. The average width of the four points is $14 \mu \mathrm{m}$ and the total length is $211 \mu \mathrm{m}$.

\subsection{Star-caved Rock Painting in Hongshanhou}

The second starhole rock carving on the red granite platform at the left side of the $50 \mathrm{~cm}$ radius below the cloud and water texture face rock painting of the Hongshan Houxingxue rock carving (codename Hmy2). 2.2 centimeters in depth and 1.5 centimeters in depth (Table 1, Figure 8). There are crystals 0.5 centimeters from the center of the celestial ridge. The angle of 90 degrees is suitable for microetching measurement. The crystal has slight attenuation, and the nine segments are accurately measured according to changes in the attenuation curve. The length is $330 \mu \mathrm{m}$ and the widths are: $15,11,17,12,17,12,11,12,15=122 / 9=$ $13.6 \mu \mathrm{m}$ (simple average), and the weighted average micro-etching width is $13.9 \approx 14 \mu \mathrm{m}$.

Micorocrystalline erosion pattern of five sites in microscopic perspective
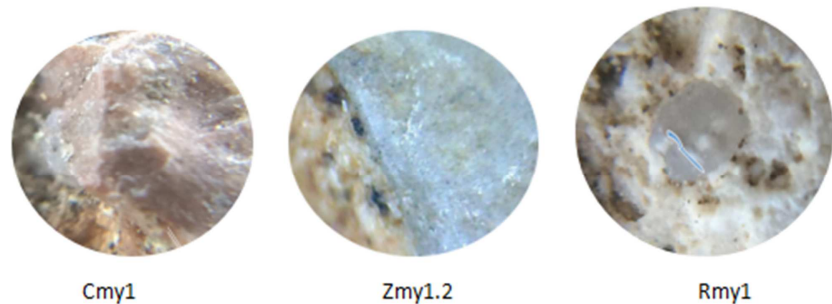

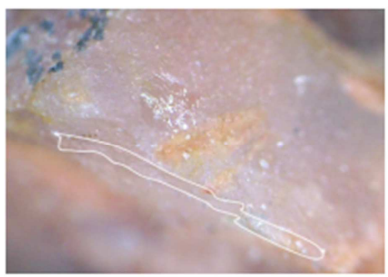

Hmy2

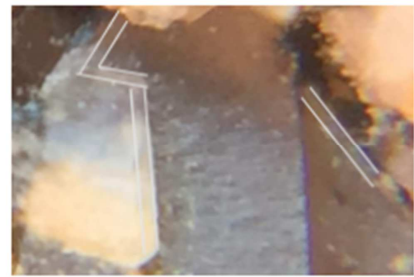

Hmy1.1/Hmy1.2/Hmy1.3/Hmy1.4
Figure 8. Phenomenon of micro-etching state of quartz crystals in five sites.

\section{Introduction to the Three Years of Quanning Road and Micro-etching Results}

Cmyl is ugly and unwitting, and the chief executive of the Yuandu Capital Administration passed away and died in the second year (1336). In the same year, he built a tomb in the archaeological village of Wudan Town, Quanning Road (now Wengniute Banner). The monument is rich in quartz crystals. Cmyl is a quartz crystal micro-corrosion sample [30, 31] (Table 1, Figure 9) in the Yuan Dynasty "All Ning Zhang first inscription".

Rmy1 Yuan Dynasty Quanning Lu Xiang Gegeji Grand 
Princess (Yuanshun Zongnü, Wu Zongmei, Renzongzi, Wenzong Mother-in-law) She was in the Yuantai Ding two years (1325) in Quanning Road (Wudan Town, Wengniute Banner) Confucianism The school built the "Jinning Lu New Confucian School Record" monument, and the figure Rmy1 is the quartz crystal micro-corrosion sample in the Yuan Dynasty's "Jinning Lu New Confucian School Record" [32] (Table 1, Figure 9).

\section{Measurement of micro- etching points in the monument of the Quan Ning Road in Yuan Dynasty}

\section{Yuan Dynasty Zhang chou Lv Monument}

\section{Yuan Dynasty}

Confucianism Monument

\section{Yuan Dynasty Zhang Ying rui Monument}

1
Zmy1.2 Zhang Yingrui, Yuanning Quanning, the chief manager of the Luwangcheng General Administration Office, and reclaiming the government of the state, Yuantong three years (1335) to build a tomb in the Pinglou Village of Wutong County, Wutongniu Town. The monument is rich in quartz crystals. Figure Cmy11.2 is a micro-corrosion sample of quartz crystals in the Yuan Dynasty's "Zhang's First Monument" $\circ$ [33] (Table 1, Figure 9).
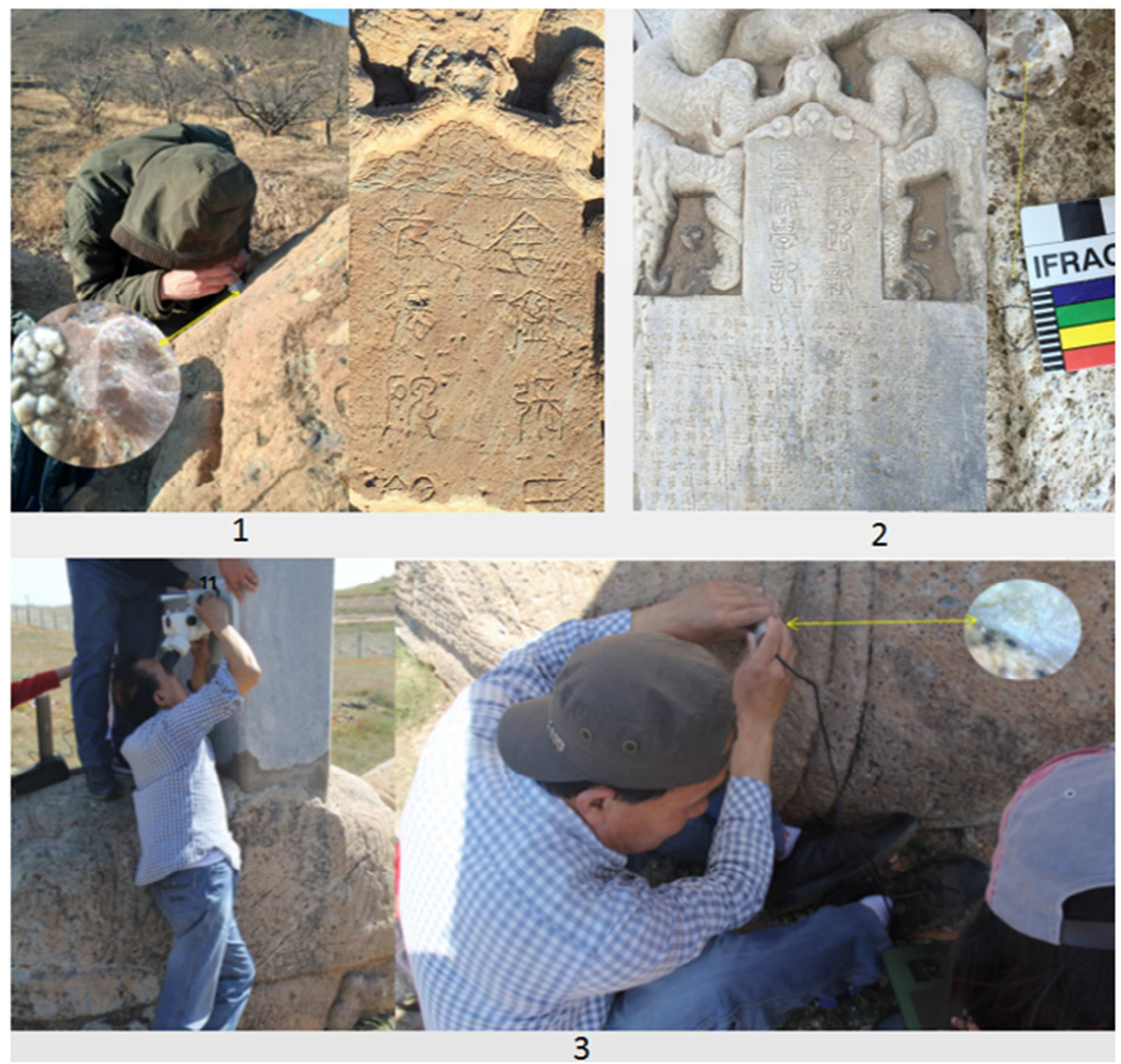

Figure 9. Measurement of micro-etching points in the monument of the Quan Ning Road in Yuan Dynasty.

The above-mentioned three monuments in the Yuan Dynasty are located in the Hongshan Cultural Area, and are less than 100 kilometers from Hongshan Later Rock Art. The natural geography and climatic conditions are the same, and the measurement data are comparable. To this end, we calculated the quartz micro-corrosion calibration coefficients (Zmy1.2, Cmy, Rmy, Table 1) at the three sites of Quanyin
Road in the Yuan Dynasty to be $2.8 \mu \mathrm{m} / \mathrm{ka}$. The face rock painting production time is E5000, (Note: "E" here, ie, erosion, said from the figure of the data of this year's figure 10); Hongshan Houxingxue rock painting age E5000 $+1071 /-1071$, the age range is 6071 - Between 3929 (Figure 11), the age of post-Hongshan rock painting belonged to the late period of the Red Mountain Culture.

Table 1. Five places in Hongshan Cultural District Sample micro-etching crystal test table.

\begin{tabular}{|c|c|c|c|c|c|c|c|c|}
\hline 1 Code & 2 Test the venue & $\begin{array}{l}3 \text { Rock } \\
\text { carving name }\end{array}$ & 4 position & 5 Oriented & 6 Angle & $\begin{array}{l}7 \text { Weighted } \\
\text { average width }\end{array}$ & 8 length & 9 AD/EPOCH \\
\hline Zmy1.1 & ZYR & Stone turtle & Front neck & Zhengxi & 90 & 1.91 & 46 & $1335 / 682$ \\
\hline Zmy1.2 & ZYR & Stone turtle & Front neck & Zhengxi & 90 & 1.91 & 66 & $1335 / 682$ \\
\hline Rmy & CFI & Right abdome & Back & Zhengxi & 90 & 1.94 & 65 & $1325 / 692$ \\
\hline Hmy1.1 & $\mathrm{HSH}$ & Human face & Bottom right & Zhengxi & 90 & 14.0 & 56 & $\mathrm{E} / 5000$ \\
\hline Hmy1.2 & $\mathrm{HSH}$ & Human face & Bottom right & Zhengxi & 90 & 14.0 & 87 & $\mathrm{E} / 5000$ \\
\hline Hmy1.4 & $\mathrm{HSH}$ & Human face & Bottom right & Zhengxi & 90 & 14.0 & 211 & $\mathrm{E} / 5000$ \\
\hline Hmy2 & $\mathrm{HSH}$ & Star cave & Zhengnan & Zhengnan & 90 & 14.0 & 264 & E5000+/-1071 \\
\hline
\end{tabular}




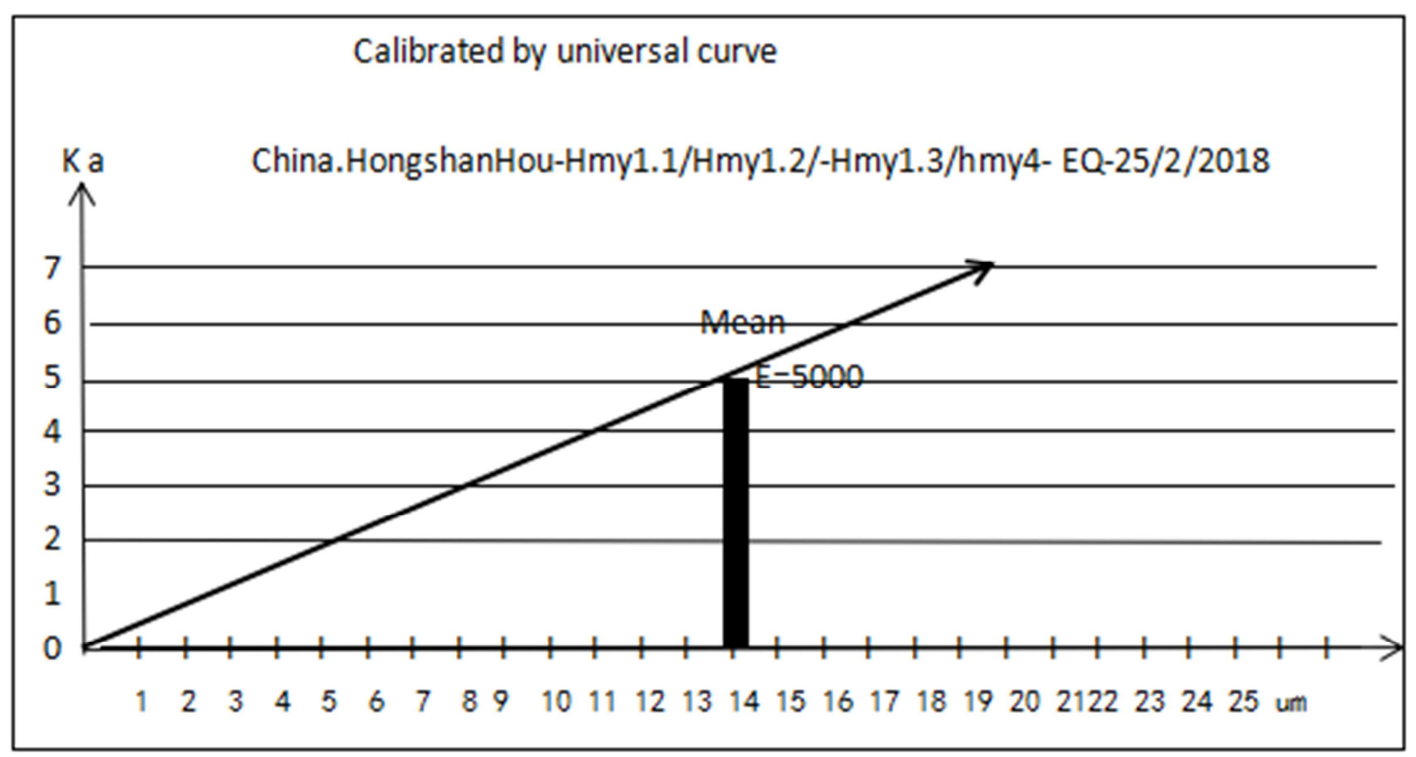

Figure 10. Calibration curve of quartz crystal micro-decay with water-cut human face image.

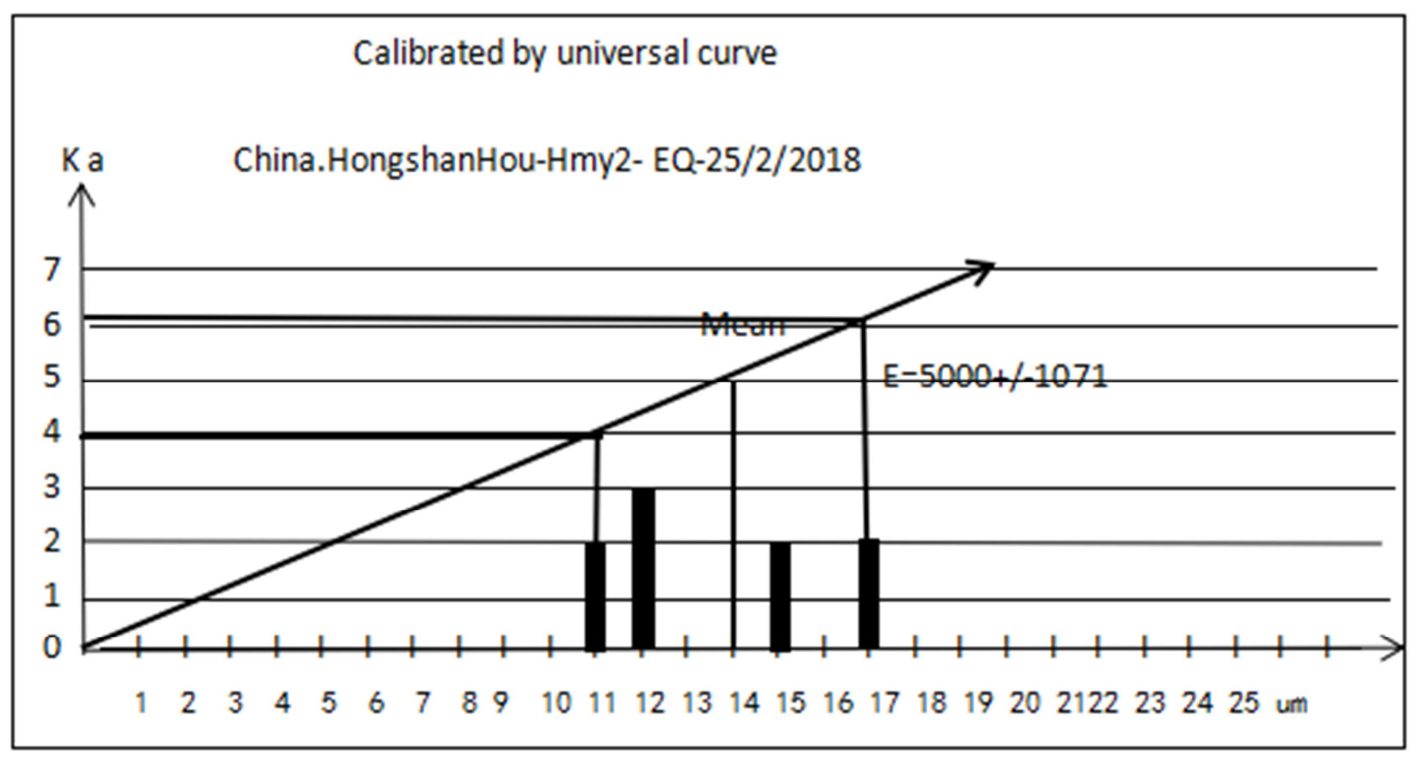

Figure 11. Starhole rock inscription Quartz crystal micro-decay calibration curve.

\section{Conclusion}

The cloud and water texture human Face Rock Paintings are distributed in the upper reaches of the Lao Ha River. According to the rock art materials published in the world and rock art materials provided by the international rock art website, it is currently verified that in the world, the cloud and water texture human Face Rock Paintings in Xiang Yan Shi is in three shops and red. There are only three in the back of the mountain, and it is formed on the Yin River, the Yingiin River, and the Laohe River. It belongs to a unique form of rock painting in northern China. The Shuiyun lithograph is not isolated. It is also linked to the star caves. The celestial caves should be related to the sky constellation at that time. The silhouette-free, water-sweeping face of the
Shuiyun pattern is combined with the sky stars to form a scroll between the heavens and the earth, especially on the steeply-defined stone cliffs behind the Red Hill. The composition of the stars is not simple and arbitrary. The whole figure contains secrets. It is to pray for heaven and earth's spirits to spill nectar on the world's rain, or to pray for the gods engraved on the banks of the river to bless this land of hot people from flooding. This secret There is no conclusion yet. However, we analyze from another aspect of rock art. This special cultural form symbol contains the initial form of the word "Cloud, Water, and Chuan" in Oracle. This unique literary character structure plays an important role in the formation of the initial writing, the role of the divine grid, the evolution and propagation of decorative graphics, and the study of ancient constellation shape and change. The age of 
petroglyphs was comprehensively measured by scientific methods such as micro-etching of crystals. It was about 5,000 years ago and it belongs to the late period of the Neolithic Hongshan Culture.

\section{Fund Project}

Inner Mongolia Social Science Planning Project 2018 (Project No.: 2018ZJD021).

\section{References}

[1] [Australia] Robert G. Bernard Jr.: Rock Art, New Delhi, India, Aryan International Book Press.

[2] Tang Huisheng, Kumar, Jin Anni, Wu Jiacai, Robert: The2015 Rock Art Miss Ions In China Rock Art Research.

[3] Wu Jiacai: "Animal Warriors Petroglyphs in the Battle of the Xiongnu and Donghu in the Damo Banner, Inner Mongolia," China Heritage News.

[4] [Swiss] Cecilia Lindkvist: "Kingdom of Chinese Characters", Life, Reading, New Knowledge, Sanlian Bookstore.

[5] [de] Karte Yemar: The Survey of Rock Art Remains in Pakistan, published by the German Institute of Anthropology.

[6] Xinjiang Cultural Relic Bureau: "Xinjiang Rock Art", Science Press.

[7] Tibetan Heritage Committee: Tibetan Rock Paintings, Sichuan People's Publishing House.

[8] [India] Giriraj Kumar: Indian rock paintings sharaa Publishing House.

[9] [Russian] Ekaterina De Wright: The Chronicles of the Altai Stele, Moscow IA RAN Press.

[10] Gao Yerong: "Wanshan Rock Sculpture", published by Taiwan Cultural Assets Bureau.

[11] [Australia] Robert Rotton: Australian Rock Paintings, Cambridge University Press, United Kingdom, [Russian] Ekaterina Devlett: "Russian Rock Art World", published by Institute of Archaeology, Russian Academy of Sciences.

[12] [America] Bill Markrona: Colorado Rock Art, Misras Press.

[13] Wen Yu, Chief Editor: Complete Works of Western Regions Rock Paintings, Xinjiang Fine Arts Photography Press.
[14] Zhu Lifeng: "Northern Human Face Rock Paintings from the Perspective of the Pacific Rim", China Social Science Pres.

[15] [Day] Yoshida Shinkio: "Japanese Rock Art", published by the Japan Society of Rock Art.

[16] Cheng Faguang: The World Petrographs, Tianjin Publishing and Media Group.

[17] [United States] Engels. Kwai Yin Lan: "The United States Great Basin Petroglyphs," Nevada University Press.

[18] [United States] David Waitley: The Handbook of Rock Art, University of Utah Press.

[19] [America] James Cassar: "Columbia Indian Rock Art", Washington University Press.

[20] [United States] Warms. Swager: The Ohio Rock Art, Ohio University Press.

[21] [United States] Edward Le Nick: "American Indian Rock Art", New England State University Press, [America] James Cassar: Plain Indian Rock Art, Washington University Press, [United States] Polly Shafma: Rock Paintings of New Mexico, New Mexico State University Press, [U.S.] Mishel Haward: "Carbon Rock Paintings," University Press, Alabama, USA.

[22] [France] Jean. Clautz: "World Rock Paintings", Getty Publishing, Los Angeles, CA.

[23] [meaning] Emanuel Anati: "The Origin of Art", Renmin University of China Press.

[24] Guo Zhizhong, Hu Chunbai; "Relics of Xiajiadian Culture in the Lower Stratum of Sanzuo City, Inner Mongolia", "Archaeology".

[25] [Days] Bintian Farming: Remains of the First Historical Site of Chifeng Hongshan in Rehe Province, East Asia Archaeological Society. Showa Thirteen Years (1938) September.

[26] Li Junyi; Interpretation of Chinese in the Yuan Dynasty's "Inscriptions on Zhang Xian's First Moral Inscription" in the Yuan Dynasty, "Northern Cultural Relics".

[27] Ga ridi; Interpretation of Chinese in the Yuan Dynasty's "Inscriptions on Zhang Xian's First Moral Inscription" in the Yuan Dynasty, "Northern Cultural Relics", Mongolia.

[28] Li Junyi: "Research on the New Confucianism in Yuanning Road in Yuan Dynasty", "Northern Cultural Relics",

[29] Wengniute Banner, Inner Mongolia", "Relics". 